\title{
BRAF, C-KIT, and NRAS mutations correlated with different clinicopathological features: an analysis of 691 melanoma patients from a single center
}

\author{
Min Ren ${ }^{1,2 \#}$, Jing Zhang ${ }^{1,2 \#}$, Yunyi Kong ${ }^{1,2}$, Qianming Bai ${ }^{1,2}$, Peng Qi ${ }^{1,2}$, Ling Zhang ${ }^{1,2}$, Qian Wang ${ }^{1,2}$, \\ Xiaoyan Zhou ${ }^{1,2}$, Yong Chen ${ }^{2,3}$, Xiaoli Zhu ${ }^{1,2}$ \\ ${ }^{1}$ Department of Pathology, Fudan University Shanghai Cancer Center, Shanghai, China; ${ }^{2}$ Department of Oncology, Shanghai Medical College, \\ Fudan University, Shanghai, China; ${ }^{3}$ Department of Musculoskeletal Oncology, Fudan University Shanghai Cancer Center, Shanghai, China \\ Contributions: (I) Conception and design: X Zhu, X Zhou; (II) Administrative support: X Zhou, Y Chen; (III) Provision of study materials or patients: \\ X Zhu, M Ren, J Zhang; (IV) Collection and assembly of data: M Ren, J Zhang, Q Bai, P Qi, L Zhang, Q Wang; (V) Data analysis and interpretation: \\ M Ren, J Zhang; (VI) Manuscript writing: All authors; (VII) Final approval of manuscript: All authors. \\ \#These authors contributed equally to this work. \\ Correspondence to: Xiaoli Zhu. Department of Pathology, Fudan University Shanghai Cancer Center, 270 Dong'an Road, Shanghai 200032, China. \\ Email: shhzx122@126.com; Yong Chen. Department of Musculoskeletal Oncology, Fudan University Shanghai Cancer Center, 270 Dong'an Road, \\ Shanghai 200032, China. Email: chenyong@fudan.edu.cn; Xiaoyan Zhou. Department of Pathology, Fudan University Shanghai Cancer Center, 270 \\ Dong'an Road, Shanghai 200032, China. Email: xyzhou100@163.com.
}

Background: Discrepancies in genetic alterations found in melanoma are conspicuous between different ethnic groups. With the approval of BRAF- and MEK-targeted inhibitors in China, it is necessary to further elucidate the landscape of gene mutation in Chinese melanoma patients.

Methods: The frequency and distribution of $B R A F, C-K I T$, and NRAS mutations in 691 melanoma patients was determined, and the statistical significance of correlations between different gene mutations and clinicopathological features was analyzed.

Results: Among a total of 691 patients, $B R A F$ mutation was found in 166 patients $(24.0 \%)$, and V600E was the prominent genetic alteration $(145 / 166,87.3 \%)$. Statistical analyses showed that younger patients $(<60)$ had a higher $B R A F$ mutation rate than older patients $(\geq 60, \mathrm{P}=0.000)$, and the frequency of $B R A F$ mutation was more likely to be lower in patients with the following: melanoma located in an extremity $(\mathrm{P}=0.000)$, acral-lentiginous melanoma subtype $(\mathrm{P}=0.000)$, thinner melanoma thickness $(\mathrm{P}=0.047)$, and no ulceration $(\mathrm{P}=0.030)$. The frequency of NRAS mutation was $12.6 \%(38 / 302)$, and primarily involved codon 61 in exon 3 and codon 12 in exon 2. Mutation of C-KIT was detected in 65 patients (9.4\%), and the most common site of mutations was L576 in exon 11 (29/65, 44.6\%). Patients with NRAS or C-KIT mutation had higher Clark level ( $\mathrm{P}=0.035$ and 0.047 , respectively) and were more likely to have melanoma located in an extremity $(\mathrm{P}=0.003$ and 0.009 , respectively) than those without such mutation. The concordance of gene mutations between paired primary and metastatic lesions was 89.6\% (60/67), and visceral metastases showed the highest distribution of gene mutations versus primary melanomas (100.0\%) compared with lymph nodes $(90.9 \%)$ and cutaneous metastases $(83.3 \%)$.

Conclusions: In this large cohort of Chinese melanoma patients, the frequencies of BRAF and NRAS mutations were lower than those observed in Caucasian cohorts, but the clinicopathological features of $B R A F, C-K I T$, and NRAS mutation were consistent. Paired primary and metastatic lesions showed high concordance of gene mutations.

Keywords: Melanoma; BRAF; C-KIT; NRAS; mutation

Submitted Aug 13, 2021. Accepted for publication Nov 23, 2021.

doi: $10.21037 /$ atm-21-4235

View this article at: https://dx.doi.org/10.21037/atm-21-4235 


\section{Introduction}

Melanoma is an extremely aggressive type of cancer with a high tumor mutation burden. It is classified into four genomic subtypes based on the pattern of the most prevalent significantly mutated genes: mutant $B R A F$, mutant $R A S$, mutant NF1, and Triple-WT (wild-type), where enrichment of KIT mutations is characteristic of the wild-type $(1,2)$. With the successful approval of BRAF and MEK dual-targeted inhibitors in China, more melanoma patients have the opportunity to receive accurate and effective targeted therapy. However, there are remarkable discrepancies in disease characteristics of melanoma between different ethnic groups (3). Compared with that observed in western Caucasian populations (34.4 per 100,000 men and 20.9 per 100,000 women), the incidence of melanoma is relatively low in China (about 0.5 per 100,000), and clear differences in the predominant histological subtypes as well as corresponding genetic alterations are also apparent $(4,5)$. Therefore, there is significant clinical value in further exploration of the prevalence of mutation characteristics in Chinese melanoma patients. Our study aimed to retrospectively analyze the prevalence and relevance of pathogenetic mutations in $B R A F, C-K I T$, and NRAS in a single-institution series of 691 melanoma patients to provide some potential references for targeted treatments in China. We present the following article in accordance with the MDAR reporting checklist (available at https://atm. amegroups.com/article/view/10.21037/atm-21-4235/rc).

\section{Methods}

\section{Patients and samples}

The study included 691 patients who were diagnosed with melanoma from 2013 to 2019 in the Fudan University Shanghai Cancer Center. All participants had their diagnosis confirmed by pathology. Complete sets of clinical information (gender, age of diagnosis, primary/metastatic/ unknown melanoma, tumor location) and histological features (histological subtype of melanoma, Breslow thickness, Clark level, ulceration and status of lymph nodes at diagnosis) for each participant were obtained. Mutation analyses of BRAF (exon 15) and C-KIT (exon 9, 11, 13 and 17) were performed in all 691 participants, and mutation analysis of NRAS (exon 2, 3, and 4) was performed in 302 participants. The study was conducted in accordance with the Declaration of Helsinki (as revised in 2013). The study was approved by the Institutional Ethics Committee at
Fudan University Shanghai Cancer Center (050432-4-2108) and individual consent for this retrospective analysis was waived.

\section{DNA extraction}

Genomic DNA was extracted from 4-5 $\mu \mathrm{m}$ unstained serial formalin-fixed paraffin-embedded (FFPE) sections. The standard xylene-phenol protocol was used to dissolve the paraffin, and the tissue specimens were digested with proteinase K. Genomic DNA was extracted using a QIAamp DNA extraction kit (Qiagen, Hilden, Germany) according to the manufacturer's instructions. The concentration and quality of DNA were determined on a NanoDrop spectrophotometer (ND-1000, Thermo Fisher Scientific, Wilmington, DE, USA).

\section{Direct sequencing of BRAF, C-KIT, and NRAS genes}

Polymerase chain reaction (PCR) amplification and direct sequencing of exon 15 of the $B R A F$ gene, exons 9, 11, 13, and 17 of the $C$-KIT gene, and exons 2, 3, and 4 of the $N R A S$ gene were performed. The primer sequences of $B R A F, C-K I T$, and NRAS are listed in Table S1. The PCR conditions were as follows: $94{ }^{\circ} \mathrm{C}$ for 10 minutes, then 38 cycles of denaturing at $94{ }^{\circ} \mathrm{C}$ for 45 seconds, annealing at $60{ }^{\circ} \mathrm{C}$ for 45 seconds, extension at $72{ }^{\circ} \mathrm{C}$ for 45 seconds, and final extension at $72{ }^{\circ} \mathrm{C}$ for 7 minutes. The PCR products were purified using a QIAquick gel extraction kit (Qiagen) and were used to prepare sequencing reactions. Sequencing was performed with the Big Dye Terminator Cycle Sequencing Ready Reaction Kit (Applied Biosystems, Foster City, CA, USA) and the following PCR conditions: $94{ }^{\circ} \mathrm{C}$ for 1 minute, 24 cycles of denaturing at $94{ }^{\circ} \mathrm{C}$ for 10 seconds, annealing at $50^{\circ} \mathrm{C}$ for 5 seconds, extension at $60{ }^{\circ} \mathrm{C}$ for 1 minute, and final extension at $72{ }^{\circ} \mathrm{C}$ for 5 minutes. Sequenced PCR products were purified and all mutations were confirmed by bidirectional sequencing on an ABI 3500 genetic analyzer (Applied Biosystems, Foster City, CA, USA).

\section{Statistical analysis}

Chi-squared or Fisher's exact test were used to compare the frequencies of gene mutations for different clinicopathological features. All statistical analyses were performed with SPSS 22.0 software (IBM Corp., Chicago, IL, USA) and a $\mathrm{P}$ value $<0.05$ (2-sided) was considered 
statistically significant.

\section{Results}

\section{Clinicopatbological characteristics}

A total of 691 Chinese patients were included in our cohort, and there were 480 primary, 87 metastatic, 57 unknown primary (including melanomas only detected in lymph nodes, liver, brain, etc.), and 67 paired primary and metastatic lesions. The age of participants ranged from 20 to 89 years (median 60 years), and the gender distribution was $47.2 \%$ male and $52.8 \%$ female. Among the 547 primary patients, there were 361 cutaneous $(66.0 \%)$ and 186 mucosal (34.0\%) melanomas. Among the 361 primary cutaneous melanomas, 18 were located in the head and neck (5.0\%), 56 in the trunk (15.5\%), 51 in limbs (14.1\%), and 236 in an extremity (65.4\%). Among the 186 mucosal melanomas, the primary site was the gastrointestinal tract in 65 patients (34.9\%), the respiratory tract in 53 patients $(28.5 \%)$, the urogenital tract in 63 patients $(33.9 \%)$, and the conjunctiva in 5 patients $(2.7 \%)$. Furthermore, there were a high proportion of female patients in the mucosal melanoma cohort due to the number of patients with tumors in the female genital tract. Finally, 109 primary patients (19.9\%) presented with lymph node metastasis at diagnosis.

Among the 361 primary cutaneous melanomas, acrallentiginous melanoma (ALM; $\mathrm{n}=212 ; 58.7 \%$ ) was the most common histological subtype, followed by superficial spreading melanoma (SSM; $\mathrm{n}=91 ; 25.2 \%)$, nodular melanoma (NM; $\mathrm{n}=50 ; 13.9 \%)$, lentigo maligna melanoma (LMM; $\mathrm{n}=5 ; 1.4 \%)$, and others $(\mathrm{n}=3 ; 0.8 \%)$. The median Breslow thickness of all lesions was $3.8 \mathrm{~mm}$. Ulceration was found in 110 patients (30.5\%). Details of clinical and pathological characteristics in the cutaneous and mucosal melanoma groups are presented in Table 1.

\section{BRAF, C-KIT, and NRAS mutations in melanoma}

Among 691 melanoma patients, 166 exhibited BRAF exon 15 mutations $(24.0 \%$, Table 1$)$. The most common site of mutation was codon $600(\mathrm{n}=154,94.8 \%)$, and V600E was the most prominent genetic alteration $(\mathrm{n}=145,87.3 \%)$. We detected C-KIT mutations in 65 of 691 participants $(9.4 \%$, Table 1), mainly occurring in exon 11 and 13 . Furthermore, L576 in exon 11 was the predominant mutated site in C-KIT mutation ( $\mathrm{n}=29,44.6 \%)$, and the common amino acid changes were L576P in exon $11(\mathrm{n}=25)$ and K642E in exon $13(\mathrm{n}=9)$. Among 302 participants who underwent NRAS mutation testing, 38 (12.6\%) had a mutation in NRAS gene. The most common sites of mutation were codon 61 in exon $3(63.2 \%, 24 / 38)$ and codon 12 in exon 2 (23.7\%, 9/38). Common amino acid changes were Q61R > Q61K > Q61H in codon 61 of exon 3 and G12D > G12R > G12C in codon 12 of exon 2, but there was no mutation in exon 4. Representative figures of BRAF, C-KIT, and NRAS mutations are presented in Figure S1.

This study included 4 patients with simultaneous $B R A F$ and $C-K I T$ gene mutations (S614P in BRAF plus 570_576 deletion in C-KIT exon 11, G593D in BRAF plus 570_576 deletion in $C$-KIT exon $11, \mathrm{~V} 600 \mathrm{E}$ in $B R A F$ plus K484R in $C-K I T$ exon 9, and V600E in BRAF plus D820Y in C-KIT exon 17), 1 patient with mutations in both Q61R in NRAS exon 3 and T500A in C-KIT exon 9, and 1 patient with 2 C-KIT mutations including V560D in exon 11 and N822H in exon 17.

\section{Correlation of BRAF, C-KIT, and NRAS mutations with clinicopathological features}

In our cohort of 691 patients, the frequency of $B R A F$ mutation in younger patients $(<60)$ was statistically higher than that in older patients $(\geq 60)(31.3 \%$ vs. $17.2 \%$, $\mathrm{P}=0.000)$. There was no difference observed in $B R A F$ mutation on the basis of gender. A $B R A F$ mutation was observed in $41.4 \%$ of metastases (36/87), a significant increase in mutation frequency compared with that in primary melanoma $(111 / 547,20.3 \%, \mathrm{P}=0.000)$. In terms of primary tumor location, $B R A F$ mutation was detected in $27.7 \%(100 / 361)$ of cutaneous melanoma but in only $5.9 \%$ $(11 / 186)$ of mucosal melanoma $(\mathrm{P}=0.000)$. Unlike $B R A F$ mutation, the frequency of $C-K I T$ mutation in younger patients was lower than that in older patients $(6.2 \%$ vs. $12.4 \%, \mathrm{P}=0.006)$. Finally, there was no significant difference between C-KIT or NRAS mutation on the basis of gender, primary/metastatic, or cutaneous/mucosal melanomas.

Among the 361 cutaneous melanoma participants, those with tumors located in the head and neck, trunk, and limbs had significantly higher $B R A F$ mutation frequency than patients with tumors located in an extremity $(56.0 \%$ vs. $12.7 \%, \mathrm{P}=0.000)$. When mutations were stratified by histological subtypes, the frequency of $B R A F$ mutation in ALM was lower than that in other subtypes of melanoma ( $11.8 \%$ vs. $50.3 \%, \mathrm{P}=0.000)$. In this study, patients with $B R A F$ mutation had thicker tumors $(\mathrm{P}=0.047)$ and less ulceration $(\mathrm{P}=0.030)$ than those without BRAF mutation 
Table 1 Basic clinical and pathological information and the prevalence of mutation observed in 691 melanoma patients

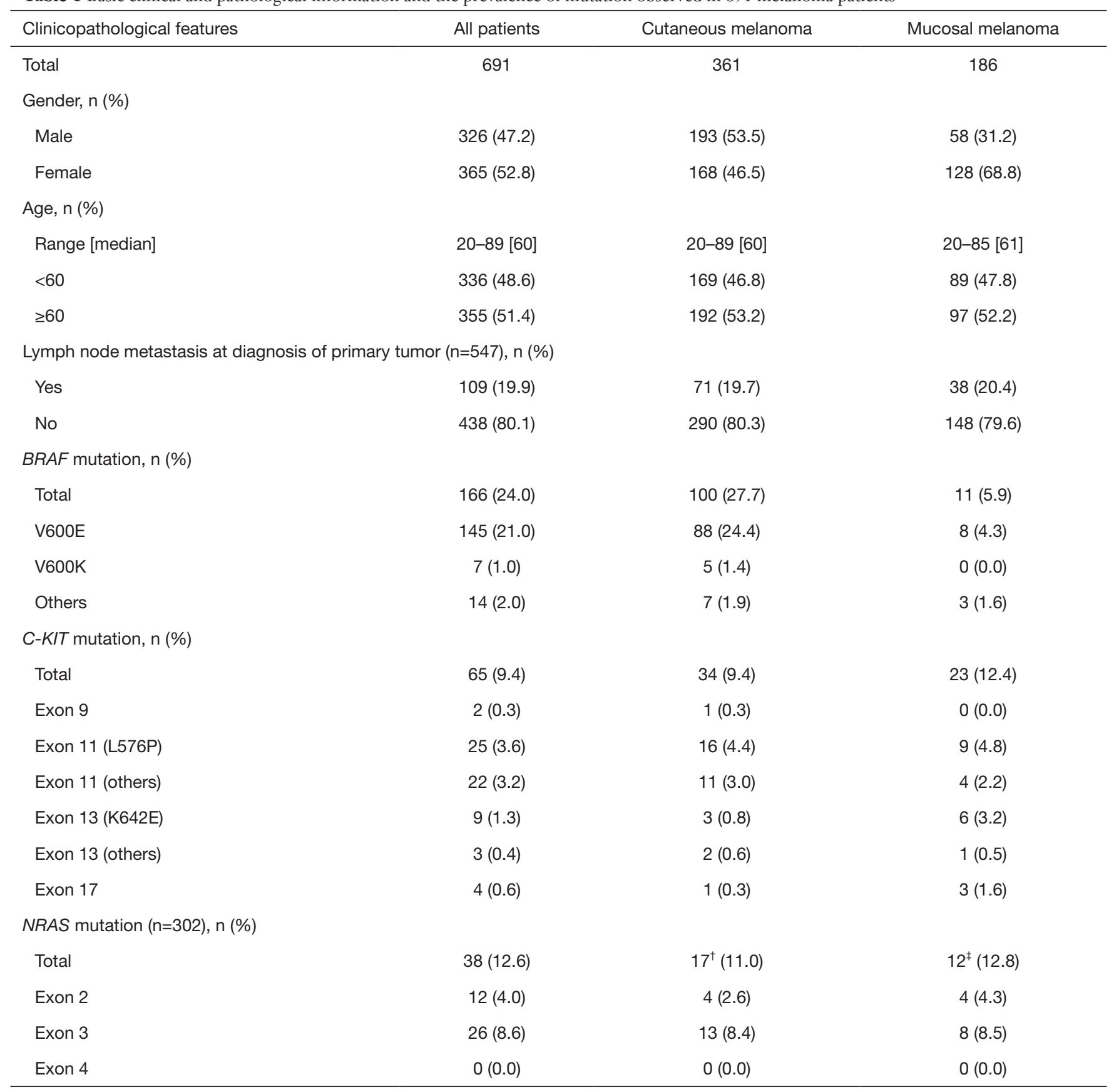

${ }^{\dagger}$, there were 155 cutaneous melanoma cases with NRAS mutation testing; ${ }^{\ddagger}$, there were 94 mucosal melanoma cases with NRAS mutation testing.

(Table 2). In addition, C-KIT and NRAS mutations were more frequently detected in participants with tumors located in an extremity $(\mathrm{P}=0.003$ and 0.009 , respectively) and higher Clark level ( $\mathrm{P}=0.035$ and 0.047 , respectively), as shown in Table 2.
In the cohort of 186 mucosal melanoma patients, different primary sites correlated with distinct features of gene mutations. The incidence of $B R A F$ mutation was higher in gastrointestinal tract mucosal melanoma than melanoma in other sites $(10.8 \%$ vs. $3.3 \%, \mathrm{P}=0.000)$ 
Table 2 Correlation of $B R A F, C-K I T$, and NRAS mutations with clinicopathological features in 361 cutaneous melanoma cases

\begin{tabular}{|c|c|c|c|c|c|c|}
\hline Clinicopathological features & \multicolumn{2}{|c|}{$B R A F$ mutation } & \multicolumn{2}{|c|}{ C-KIT mutation } & \multicolumn{2}{|c|}{ NRAS mutation } \\
\hline \multicolumn{7}{|l|}{ Gender } \\
\hline Male & $51 / 193(26.4)$ & 0.561 & 19/193 (9.8) & 0.778 & $9 / 81(11.1)$ & 0.952 \\
\hline Female & 49/168 (29.2) & & 15/168 (8.9) & & 8/74 (10.8) & \\
\hline$<60$ & 66/169 (39.1) & 0.000 & $7 / 169(4.1)$ & 0.001 & 6/69 (8.7) & 0.417 \\
\hline$\geq 60$ & $34 / 192(17.7)$ & & 27/192 (14.1) & & $11 / 86(12.8)$ & \\
\hline \multicolumn{7}{|c|}{ Location of cutaneous melanoma } \\
\hline Extremity & $30 / 236(12.7)$ & 0.000 & $30 / 236(12.7)$ & 0.003 & 16/102 (15.7) & 0.009 \\
\hline Yes & 22/71 (31.0) & 0.490 & $7 / 71(9.9)$ & 0.887 & 2/29 (6.9) & 0.741 \\
\hline No & $78 / 290(26.9)$ & & $27 / 290(9.3)$ & & 15/126 (11.9) & \\
\hline \multicolumn{7}{|c|}{ Histological type of cutaneous melanoma } \\
\hline ALM & $25 / 212(11.8)$ & 0.000 & $25 / 212(11.8)$ & 0.218 & 14/96 (14.6) & 0.066 \\
\hline Others & $75 / 149(50.3)$ & & $9 / 149(6.0)$ & & $3 / 59(5.1)$ & \\
\hline \multicolumn{7}{|c|}{ Breslow thickness of cutaneous melanoma, $\mathrm{mm}$} \\
\hline$\leq 1.0$ & $6 / 40(15.0)$ & 0.047 & $2 / 41(4.9)$ & 0.401 & $0 / 16(0.0)$ & 0.045 \\
\hline$>1.0$ & $94 / 321$ (29.3) & & $32 / 320(10.0)$ & & $17 / 139(12.2)$ & \\
\hline Absent & $78 / 251(31.1)$ & & 19/251 (7.6) & & $11 / 108(10.2)$ & \\
\hline
\end{tabular}

Italic $P$ values indicate statistical significance. ALM, acral-lentiginous melanoma; Mut, mutation.

including the respiratory tract, urogenital tract, and conjunctival melanomas. On the other hand, NRAS mutation occurred less frequently in the respiratory tract when compared to other tumor sites ( $0 \%$ vs. $20.3 \%$, $\mathrm{P}=0.003$ ) (Table 3).

\section{Concordance of mutation between paired primary and metastatic lesions}

Among 67 paired primary and metastatic lesions, there were
44 lymph nodes, 18 cutaneous metastases, and 5 visceral metastases. Mutation inconsistencies in BRAF, C-KIT, and $N R A S$ genes were found in 7 patients $(10.4 \%)$, including 4 BRAF discordance (9.1\%), 1 C-KIT discordance (2.3\%), and 2 NRAS discordance (2/39, 5.1\%). Stratified by different types of metastases, visceral metastases ( $100.0 \%$ consistency) presented the most similar distribution of $B R A F / C-K I T /$ $N R A S$ mutations $v s$. primary melanomas, followed by lymph nodes (90.9\% consistency) and cutaneous metastases (83.3\% consistency). 
Table 3 Correlation of $B R A F, C-K I T$, and NRAS mutations with clinicopathological features in 186 mucosal melanoma cases

\begin{tabular}{|c|c|c|c|c|c|c|}
\hline Clinicopathological features & \multicolumn{2}{|c|}{$B R A F$ mutation } & \multicolumn{2}{|c|}{$C-K I T$ mutation } & \multicolumn{2}{|c|}{ NRAS mutation } \\
\hline \multicolumn{7}{|l|}{ Gender } \\
\hline Male & $6 / 58(10.3)$ & 0.100 & 6/58 (10.3) & 0.573 & $3 / 34(8.8)$ & 0.526 \\
\hline Female & $5 / 128(3.9)$ & & $17 / 128(13.3)$ & & $9 / 60(15.0)$ & \\
\hline$<60$ & $5 / 89(5.6)$ & 0.870 & 9/89 (10.1) & 0.371 & $4 / 37(10.8)$ & 0.759 \\
\hline$\geq 60$ & 6/97 (6.2) & & 14/97 (14.4) & & $8 / 57(14.0)$ & \\
\hline \multicolumn{7}{|l|}{ Location of mucosal melanoma } \\
\hline Gastrointestinal tract & $7 / 65(10.8)$ & 0.000 & 9/65 (13.8) & 0.653 & $0 / 35(0.0)$ & 0.003 \\
\hline Yes & $3 / 38(7.9)$ & 0.971 & $4 / 38(10.5)$ & 0.699 & $1 / 20(5.0)$ & 0.450 \\
\hline No & $8 / 99(8.1)$ & & $19 / 148(12.8)$ & & $11 / 74(14.9)$ & \\
\hline
\end{tabular}

Italic $P$ values indicate statistical significance. Mut, mutation.

\section{Discussion}

Until now, most studies of the landscape of genomic alterations in melanoma have focused on western populations $(6,7)$, so there remains a need to fully delineate the oncogenic differences in melanoma between Caucasian and Asian populations. By conducting a large-scale singleinstitution study of genetic mutations in melanoma, we have identified particular features of BRAF, C-KIT, and NRAS mutations which may have significant clinical value for the treatment of Chinese melanoma patients. With regard to clinical characteristics, this study and previous studies on eastern populations have consistently found that melanomas in Asian patients are more likely to be located in extremities, be thicker, have a higher rate of ulceration, and be accompanied by poorer prognoses as compared to melanomas in western patients $(3,8-10)$.

The $B R A F$ gene, as the major mutated gene in melanoma, is found to be mutated in about $50-60 \%$ of western melanoma patients and associated with intermittent ultraviolet (UV) exposure $(11,12)$. However, because the most common tumor locations are sun-protected extremities and mucosa ( $70 \%$ patients), the frequency of $B R A F$ mutation is only around $20 \%$ in Asians $(8,13,14)$. Despite the relatively limited frequency of $B R A F$ mutation in the Chinese population, our results are consistent with previous findings in that the dominated position of $B R A F$ mutation is codon 600 , and more than $90 \%$ of the amino acid change is $\mathrm{V} 600 \mathrm{E}$ which could activate the function of this kinase $(8,13)$. Also consistent with previous studies, $B R A F$ mutation prevailed in younger patients $(4,15)$ but was rare in extremities and corresponding ALM subtype, confirming the UV-induced nature of $B R A F$ mutation $(4,16)$. Our study also revealed that thicker and metastatic lesions had markedly higher rates of $B R A F$ mutation than thinner and primary lesions (17-19), indicating an increase in $B R A F$ mutation as melanoma progresses. Further study is needed to investigate the role of $B R A F$ mutation in melanomagenesis and progression. Additionally, $B R A F$ mutation was found in $5.9 \%$ of mucosal melanoma patients in our cohort, but non-V600E was more common (3/11, $27.3 \%)$ compared with that in cutaneous melanoma (12/100, $12.0 \%)$. This is in agreement with the findings of Bai et al.'s study of 2,793 Chinese patients (13). Contrary to the results of a meta-analysis of mucosal melanoma (20), we found that gastrointestinal tract melanomas, rather than conjunctival melanomas, exhibited more $B R A F$ mutation, probably owing to the relatively limited number of conjunctival melanoma patients in our study.

Consistent with previous reports (13,21-23), the frequencies of $C-K I T$ and NRAS mutations in melanoma were about $8.0 \%$ and $10.4 \%$, respectively. The pattern of C-KIT mutation was dominated by L576 and K642 
(38/65, 58.5\%), and NRAS mutation was dominated by Q61, G12, and G13 (36/38, 94.7\%). In contrast to the clinicopathological features of $B R A F$ mutation, the frequencies of $C-K I T$ and NRAS mutations in older patients were higher than those in younger patients. Tumors located in an extremity and ALM subtype tended to exhibit more $C$-KIT or NRAS mutations as compared to previous reports $(12,18,21,24-26)$. Furthermore, our study found that NRAS-mutated patients showed higher Clark level and deeper Breslow thickness compared to negative patients, and C-KIT mutation was also correlated with higher Clark level. However, most of the studies conducted to date did not reveal a significant correlation between $C-K I T$ or NRAS mutation with level of tumor invasion $(9,10,16,18,27)$, and Thomas et al. (28) found that NRAS mutation was associated with lower ulceration rate, which was nonsignificant in our study. Discrepancies in the clinical relevance of $C$-KIT and NRAS mutations in different studies may be related to distinct ethnic groups and sample size, and may reveal genetic information particular to the Chinese population.

Generally, hotspots for mutations in the BRAF, C-KIT, and NRAS genes, as the key molecules in the mitogenactivated protein kinase (MAPK) pathway, were mutually exclusive in melanoma (1). In our study, 6 participants simultaneously exhibited mutations in two distinct genes (BRAF, C-KIT, or NRAS), but at least 1 of these gene mutations did not correspond to a hotspot and so the biochemical and the clinical value requires further investigation. However, in the report by Bai et al. (13), 13 patients exhibited both BRAF V600E and NRAS Q61R/ $\mathrm{K}$ or G12D mutations, suggesting that we should be aware of such exceptional genetic alterations so that patients can have routine access to appropriate treatment.

Several previous studies have described the heterogeneity of genetic alterations between primary and corresponding metastatic lesions in melanoma (19,29-31). In our cohort, the concordance rates of BRAF, C-KIT, and NRAS mutations between paired primary and metastatic lesions were $94.0 \%, 97.7 \%$, and $94.9 \%$, respectively. A recent study conducted by Cormican et al. reported the concordance rate of $B R A F$ mutation in matched primary and metastatic melanoma $(52 / 53,98 \%)$ (30) to be even higher than the results in our study. However, the study conducted by Manca et al. revealed that mutational concordance in pathogenic/pathogenic-like mutations between primary and metastatic melanomas was only $76 \%$ (6), and that diverse genetic alterations could exist at different sites originating from the same lesion (32-34). Due to the size of paired primary and metastatic samples was relatively limited, the high concordance of gene mutations in paired primaries and metastases in our study needs to be further evaluated in larger cohort containing a more balanced distribution of different melanoma subtypes. The intra- and intertumor heterogeneities of gene mutations indicate that different sites and periods of lesions should all be evaluated in establishing the status of mutation, in order to avoid omitting any patients who may benefit from a targeted therapy. When considering different types of metastasis, visceral and lymph node metastases, as compared with cutaneous metastases, presented highly similar mutation status relative to the primary lesions, which was consistent with the results of Colombino et al. (29).

In conclusion, this study has shown that when compared with the mutation distribution in Caucasian cohorts, Chinese melanoma patients exhibit lower $B R A F$ mutation rates and higher $C-K I T$ and NRAS mutation rates. The common genotypes of $B R A F, C-K I T$, and NRAS mutations, however, were found to be similar. The diverse clinicopathological characteristics of $B R A F$, $C-K I T$, and NRAS mutations delineated the particular genomic landscape of Chinese melanoma patients. The discrepancies in mutation status between primary and metastatic lesions highlight a need to fully comprehend the genetic background of patients who may otherwise miss out targeted treatment. Further studies using more comprehensive and accurate detection methods, including next generation sequencing and whole exome sequencing, are warranted to clarify the pathogenesis and explore new therapeutic targets to improve treatment of melanoma in China.

\section{Acknowledgments}

Funding: This work was supported by the Innovation Group Project of Shanghai Municipal Health Commission (2019CXJQ03), the Shanghai Science and technology development fund (19MC1911000), and the Shanghai Municipal Key Clinical Specialty (shslczdzk01301).

\section{Footnote}

Reporting Checklist: The authors have completed the MDAR reporting checklist. Available at https://atm.amegroups. com/article/view/10.21037/atm-21-4235/rc

Data Sharing Statement: Available at https://atm.amegroups. 


\section{Page 8 of 9}

com/article/view/10.21037/atm-21-4235/dss

Peer Review File: Available at https://atm.amegroups.com/ article/view/10.21037/atm-21-4235/prf

Conflicts of Interest: All authors have completed the ICMJE uniform disclosure form (available at https://atm.amegroups. com/article/view/10.21037/atm-21-4235/coif). All authors report that this work was supported by the Innovation Group Project of Shanghai Municipal Health Commission (2019CXJQ03), the Shanghai Science and technology development fund (19MC1911000), and the Shanghai Municipal Key Clinical Specialty (shslczdzk01301). The authors have no other conflicts of interest to declare.

Ethical Statement: The authors are accountable for all aspects of the work in ensuring that questions related to the accuracy or integrity of any part of the work are appropriately investigated and resolved. The study was conducted in accordance with the Declaration of Helsinki (as revised in 2013). The study was approved by the Institutional Ethics Committee at Fudan University Shanghai Cancer Center (050432-4-2108) and individual consent for this retrospective analysis was waived.

Open Access Statement: This is an Open Access article distributed in accordance with the Creative Commons Attribution-NonCommercial-NoDerivs 4.0 International License (CC BY-NC-ND 4.0), which permits the noncommercial replication and distribution of the article with the strict proviso that no changes or edits are made and the original work is properly cited (including links to both the formal publication through the relevant DOI and the license). See: https://creativecommons.org/licenses/by-nc-nd/4.0/.

\section{References}

1. Cancer Genome Atlas Network. Genomic Classification of Cutaneous Melanoma. Cell 2015;161:1681-96.

2. Leichsenring J, Stögbauer F, Volckmar AL, et al. Genetic profiling of melanoma in routine diagnostics: assay performance and molecular characteristics in a consecutive series of 274 cases. Pathology 2018;50:703-10.

3. Lv J, Dai B, Kong Y, et al. Acral Melanoma in Chinese: A Clinicopathological and Prognostic Study of 142 cases. Sci Rep 2016;6:31432.

4. Kim SY, Kim SN, Hahn HJ, et al. Metaanalysis of BRAF mutations and clinicopathologic characteristics in primary
Ren et al. Mutations and clinicopathological features of melanoma

melanoma. J Am Acad Dermatol 2015;72:1036-46.e2.

5. Dimitriou F, Krattinger R, Ramelyte E, et al. The World of Melanoma: Epidemiologic, Genetic, and Anatomic Differences of Melanoma Across the Globe. Curr Oncol Rep 2018;20:87.

6. Manca A, Paliogiannis P, Colombino M, et al. Mutational concordance between primary and metastatic melanoma: a next-generation sequencing approach. J Transl Med 2019;17:289.

7. Clarke LE, Flake DD 2nd, Busam K, et al. An independent validation of a gene expression signature to differentiate malignant melanoma from benign melanocytic nevi. Cancer 2017;123:617-28.

8. Si L, Kong Y, Xu X, et al. Prevalence of BRAF V600E mutation in Chinese melanoma patients: large scale analysis of BRAF and NRAS mutations in a 432-case cohort. Eur J Cancer 2012;48:94-100.

9. Jin SA, Chun SM, Choi YD, et al. BRAF mutations and KIT aberrations and their clinicopathological correlation in 202 Korean melanomas. J Invest Dermatol 2013;133:579-82.

10. Kong Y, Si L, Zhu Y, et al. Large-scale analysis of KIT aberrations in Chinese patients with melanoma. Clin Cancer Res 2011;17:1684-91.

11. Saroufim M, Habib R, Karram S, et al. BRAF analysis on a spectrum of melanocytic neoplasms: an epidemiological study across differing UV regions. Am J Dermatopathol 2014;36:68-73.

12. Moltara ME, Novakovic S, Boc M, et al. Prevalence of BRAF, NRAS and c-KIT mutations in Slovenian patients with advanced melanoma. Radiol Oncol 2018;52:289-95.

13. Bai X, Kong Y, Chi Z, et al. MAPK Pathway and TERT Promoter Gene Mutation Pattern and Its Prognostic Value in Melanoma Patients: A Retrospective Study of 2,793 Cases. Clin Cancer Res 2017;23:6120-7.

14. Yamazaki N, Tanaka R, Tsutsumida A, et al. BRAF V600 mutations and pathological features in Japanese melanoma patients. Melanoma Res 2015;25:9-14.

15. Long GV, Menzies AM, Nagrial AM, et al. Prognostic and clinicopathologic associations of oncogenic BRAF in metastatic melanoma. J Clin Oncol 2011;29:1239-46.

16. Lee JH, Choi JW, Kim YS. Frequencies of BRAF and NRAS mutations are different in histological types and sites of origin of cutaneous melanoma: a meta-analysis. $\mathrm{Br}$ J Dermatol 2011;164:776-84.

17. Hugdahl E, Kalvenes MB, Puntervoll HE, et al. BRAFV600E expression in primary nodular melanoma is associated with aggressive tumour features and reduced 
survival. Br J Cancer 2016;114:801-8.

18. Ponti G, Manfredini M, Greco S, et al. BRAF, NRAS and C-KIT Advanced Melanoma: Clinico-pathological Features, Targeted-Therapy Strategies and Survival. Anticancer Res 2017;37:7043-8.

19. Heinzerling L, Baiter M, Kühnapfel S, et al. Mutation landscape in melanoma patients clinical implications of heterogeneity of BRAF mutations. Br J Cancer 2013;109:2833-41.

20. Mikkelsen LH, Larsen AC, von Buchwald C, et al. Mucosal malignant melanoma - a clinical, oncological, pathological and genetic survey. APMIS 2016;124:475-86.

21. Sakaizawa K, Ashida A, Uchiyama A, et al. Clinical characteristics associated with BRAF, NRAS and KIT mutations in Japanese melanoma patients. J Dermatol Sci 2015;80:33-7.

22. Sheen YS, Liao YH, Liau JY, et al. Prevalence of BRAF and NRAS mutations in cutaneous melanoma patients in Taiwan. J Formos Med Assoc 2016;115:121-7.

23. Dumaz N, André J, Sadoux A, et al. Driver KIT mutations in melanoma cluster in four hotspots. Melanoma Res 2015;25:88-90.

24. Ellerhorst JA, Greene VR, Ekmekcioglu S, et al. Clinical correlates of NRAS and BRAF mutations in primary human melanoma. Clin Cancer Res 2011;17:229-35.

25. Gong HZ, Zheng HY, Li J. The clinical significance of KIT mutations in melanoma: a meta-analysis. Melanoma Res 2018;28:259-70.

26. Heppt MV, Siepmann T, Engel J, et al. Prognostic significance of BRAF and NRAS mutations in melanoma: a German study from routine care. BMC Cancer 2017;17:536.

27. Oyama S, Funasaka Y, Watanabe A, et al. BRAF, KIT and

Cite this article as: Ren M, Zhang J, Kong Y, Bai Q, Qi P, Zhang L, Wang Q, Zhou X, Chen Y, Zhu X. BRAF, C-KIT, and $N R A S$ mutations correlated with different clinicopathological features: an analysis of 691 melanoma patients from a single center. Ann Transl Med 2022;10(2):31. doi: 10.21037/atm-214235
NRAS mutations and expression of c-KIT, phosphorylated extracellular signal-regulated kinase and phosphorylated AKT in Japanese melanoma patients. J Dermatol 2015;42:477-84.

28. Thomas NE, Edmiston SN, Alexander A, et al. Association Between NRAS and BRAF Mutational Status and Melanoma-Specific Survival Among Patients With HigherRisk Primary Melanoma. JAMA Oncol 2015;1:359-68. Erratum in: JAMA Oncol 2015;1:285.

29. Colombino M, Capone M, Lissia A, et al. BRAF/ NRAS mutation frequencies among primary tumors and metastases in patients with melanoma. J Clin Oncol 2012;30:2522-9.

30. Cormican D, Kennedy C, Murphy S, et al. High concordance of BRAF mutational status in matched primary and metastatic melanoma. J Cutan Pathol 2019;46:117-22.

31. Yang S, Leone DA, Biswas A, et al. Concordance of somatic mutation profiles (BRAF,NRAS, and TERT) and tumoral PD-L1 in matched primary cutaneous and metastatic melanoma samples. Hum Pathol 2018;82:206-14.

32. Grzywa TM, Paskal W, Włodarski PK. Intratumor and Intertumor Heterogeneity in Melanoma. Transl Oncol 2017;10:956-75.

33. Yancovitz M, Litterman A, Yoon J, et al. Intra- and inter-tumor heterogeneity of BRAF(V600E))mutations in primary and metastatic melanoma. PLoS One 2012;7:e29336.

34. Kaji T, Yamasaki O, Takata M, et al. Comparative study on driver mutations in primary and metastatic melanomas at a single Japanese institute: A clue for intra- and inter-tumor heterogeneity. J Dermatol Sci 2017;85:51-7. 


\section{Supplementary}

Table S1 Primers for BRAF, C-KIT, and NRAS

\begin{tabular}{|c|c|c|c|}
\hline Gene name & Forward primer & Reverse primer & Size (bp) \\
\hline Exon 15 & 5'-GCTTGCTCTGATAGGAAAATGAG-3' & 5'-GTAACTCAGCAGCATCTCAGG-3' & 237 \\
\hline \multicolumn{4}{|l|}{ C-KIT } \\
\hline Exon 9 & 5'-TCCTAGAGTAAGCCAGGGCTT-3' & 5'-TGGTAGACAGAGCCTAAACATCC-3' & 261 \\
\hline Exon 13 & 5'-GCTTGACATCAGTTTGCCAG-3' & 5'-AAAGGCAGCTTGGACACGGCTTTA-3' & 193 \\
\hline Exon 17 & 5'-TACAAGTTAAAATGAATTTAAATGGT-3' & 5'-AAGTTGAAACTAAAAATCCTTTGC-3' & 228 \\
\hline \multicolumn{4}{|l|}{ NRAS } \\
\hline Exon 2 & 5'-CAGGTTCTTGCTGGTGTGAA-3' & 5'-CACTGGGCCTCACCTCTATG-3' & 144 \\
\hline
\end{tabular}
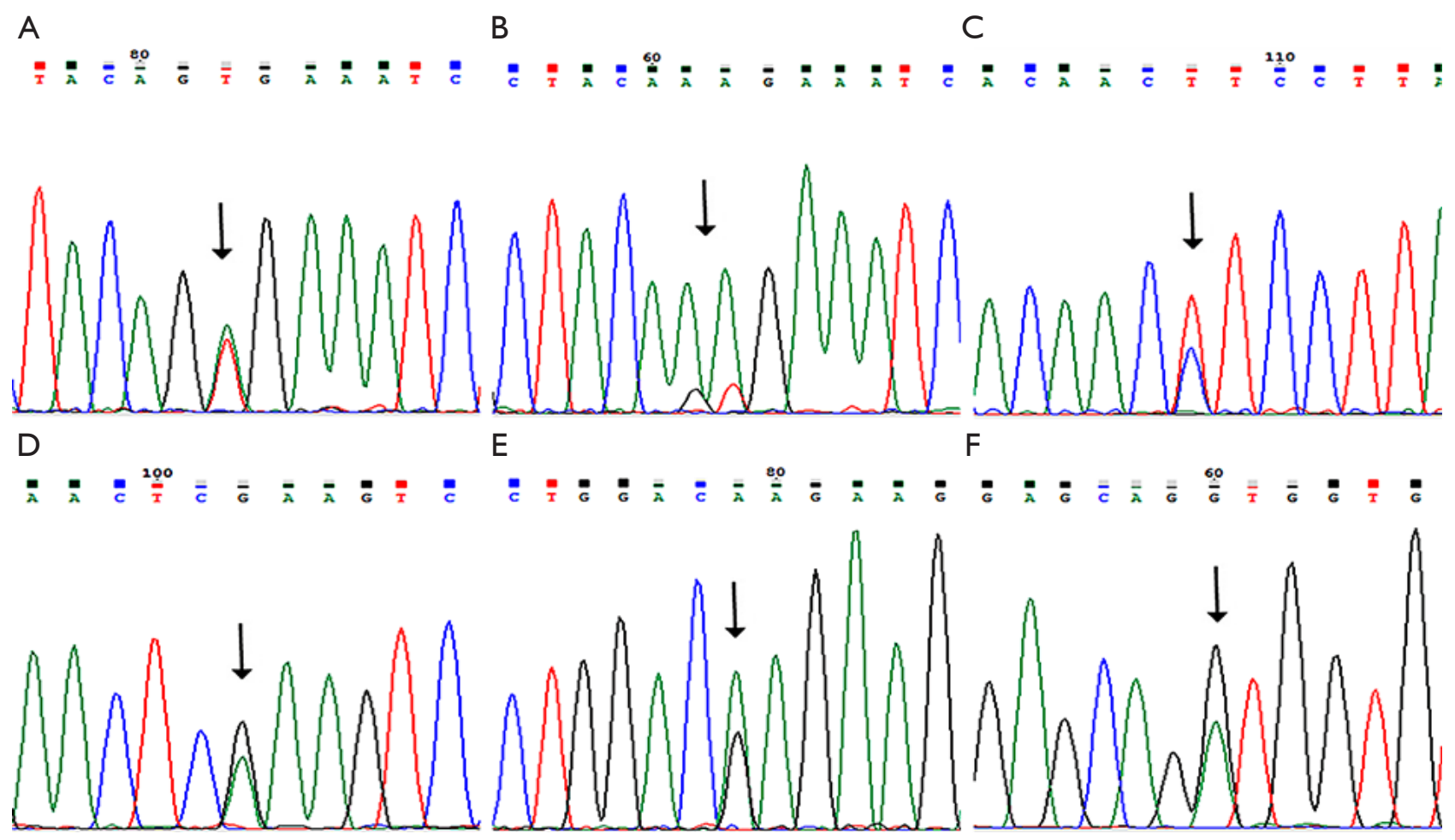

Figure S1 Representative figures of BRAF V600E (A), BRAF V600K (B), C-KIT L576P (C), C-KIT K642E (D), NRAS Q61R (E), and NRAS G12D (F). 